\title{
Analysis of a Compact and Superdirective Metamaterial-Inspired Monopole Antenna
}

\author{
Saber Dakhli, ${ }^{1,2}$ Hatem Rmili, ${ }^{3}$ Kourosh Mahdjoubi, ${ }^{4}$ \\ Jean-Marie Floc' $h$, ${ }^{2}$ and Fethi Choubani ${ }^{1}$ \\ ${ }^{1}$ Innov'Com Laboratory, SUPCOM, University of Carthage, 2083 Tunis, Tunisia \\ ${ }^{2}$ IETR, University of Rennes 1, Campus Beaulieu, Bât. 11D, No. 263, Avenue Général Leclerc, CS 74205, 35042 Rennes Cedex, France \\ ${ }^{3}$ Electrical and Computer Engineering Department, King Abdulaziz University, P.O. Box 80204, Jeddah 21589, Saudi Arabia \\ ${ }^{4}$ IETR, INSA, 20 Avenue Buttes des Coësmes, 35043 Rennes, France
}

Correspondence should be addressed to Hatem Rmili; hmrmili@kau.edu.sa

Received 15 March 2014; Revised 27 July 2014; Accepted 18 August 2014; Published 28 October 2014

Academic Editor: Zhi N. Chen

Copyright (C) 2014 Saber Dakhli et al. This is an open access article distributed under the Creative Commons Attribution License, which permits unrestricted use, distribution, and reproduction in any medium, provided the original work is properly cited.

\begin{abstract}
The directivity of a metamaterial-inspired compact $\left(0.145 \lambda_{0}\right)$ monopole antenna was investigated. The proposed structure is composed of a split-ring resonator (SRR) parasitic element placed in the vicinity of a monopole antenna. Two configurations denoted by A1 and A2 were considered depending on the position of the slot in the SRR element. By analyzing simulated and measured results, the superdirectivity ( $7.5 \mathrm{dBi}$ for $\mathrm{A} 1$ and $9 \mathrm{dBi}$ for $\mathrm{A} 2)$ of the structure was discussed. It is found that the monopoleSRR and/or ground plane-SRR coupling effects may be responsible for the superdirectivity of the structure.
\end{abstract}

\section{Introduction}

The design of compact antennas is a topic of considerable interest within the antenna community. For this reason, various miniaturization techniques [1-3] have been developed such as load of lumped elements (inductive and capacitive), use of passive and active matching circuits, new materials and composites, and metamaterials.

Recently, a new design approach based on the use of metamaterial-inspired constructs has been emerged and applied in order to improve the radiation properties of antennas [4].

Since 2007, an efficient and electrically small antenna system operating at multifrequency bands was proposed by Erentok and Ziolkowski for new generation communication systems [5]. Next, this new concept was applied to design antennas with improved performances such as miniaturization [6-9], high efficiency [10-12], enhanced bandwidth [13, $14]$, high gain $[15,16]$, and reconfigurability [17-19].

For example, a new technique inspired from a metamaterial has been proposed for the design of an ultracompact printed antenna (size around $0.178 \lambda_{0}$ ) for WLAN applications [8]. In [13], a metamaterial-inspired loading was used for the design of a compact printed dipole antenna $\left(0.294 \lambda_{0}\right.$ $\times 0.075 \lambda_{0}$ ) with enhanced bandwidth of $56 \%$.

The frequency reconfigurability of compact $\left(0.056 \lambda_{0} \times\right.$ $\left.0.047 \lambda_{0}\right)$ metamaterial-inspired antennas was also investigated in [17], where the operating frequency of a printed monopole with incorporated slot and varactor diode was tuned over the broad frequency range $1.6-2.23 \mathrm{GHz}$. High efficiency (between 80 and 91\%), in addition to radiation patterns reconfigurability, was also obtained for metamaterial inspired compact monoband antennas [18].

However, the radiation properties of miniature antennas which are highly dependent on the antenna size $[20,21]$ are usually studied in terms of gain, efficiency, and radiation quality factor and rarely with a directivity point of view. For this reason, we focus our study in this paper on the investigation of the directivity of a compact monopole metamaterialinspired antenna.

Recently, few works were interested in the superdirectivity of metamaterial-inspired compact antennas [22-27]. The 
main techniques used to realize superdirective antennas were resumed recently in [22]. These techniques are dealing with the use of high-impedance surface [23], Huygens sources $[24,25]$, lumped elements [26], and electrically small arrays [27].

In fact, the term of superdirectivity was used by Taylor in 1948 [28], with reference to the work of Riblet dealing with the maximum directivity of antennas [29].

In 1958, Harrington [30] proposed a limit for the maximum directivity $D$ of an antenna in a specific direction $\left(\theta_{0}, \varphi_{0}\right)$ by taking $N$ modes responsible for the radiation of the structure:

$$
D_{\max }\left(\theta_{0}, \varphi_{0}\right)=N^{2}+2 N
$$

Harrington used the asymptotic expansion of Hankel's functions to establish a relationship between the number of modes $N$ and the antenna size $k a$ :

$$
k a \approx N
$$

$k$ is the wave number, and $a$ is the radius of the smallest sphere enclosing the antenna.

By combining (1) and (2), we obtain

$$
D_{\max } \approx(k a)^{2}+2(k a) .
$$

With this expression, Harrington proposed the first limit of the directivity versus the antenna size. When the directivity is greater than $D_{\text {max }}$, the antenna is considered superdirective.

In this paper, we focus our study on the use of the Huygens source technique in order to improve the compactness and directivity of a monopole metamaterial-inspired antenna.

In a previous work [12], we have studied a family of efficient metamaterial-inspired antennas composed of a monopole antenna with a split-ring resonator (SRR) parasitic element. Four configurations of the antenna were studied by changing the position of the slot in the SRR loop. It has been shown that, among the four studied configurations, only two of them exhibit a high directivity.

In this work, we propose a deep study in order to analyze the superdirectivity behavior of the two previous configurations of the metamaterial-inspired compact antenna. The antenna consists of a split-ring resonator (SRR) cell placed in the near-field region of a monopole antenna in order to reduce the profile of the structure and to reach the superdirectivity $\left(D>D_{\max }\right)$. A detailed study will be presented in order to determine the origin of the obtained superdirectivity for the proposed structure.

This paper is arranged as follows. In Section 2, details on the structure design are presented. Section 3 presents both numerical analysis of the structure and its experimental validation. Finally, Section 4 gives the main concluded results.

\section{Antennas Design}

The structure of the proposed antenna is shown in Figure 1. The antenna is composed of two elements (SRR and monopole) which are printed on a dielectric substrate and

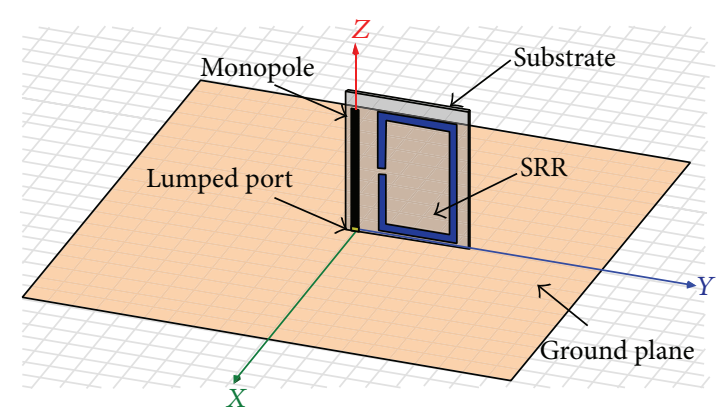

FIGURE 1: HFSS model for the antenna with configuration A1.

mounted orthogonally on a rectangular ground plane. The considered substrate is Rogers Duroid 5880 of thickness $h=$ $0.8 \mathrm{~mm}$ and relative permittivity $\varepsilon_{r}=2.2$, and the dimensions of the ground plane are $100 \times 100 \mathrm{~mm}^{2}$.

The printed monopole of length $L_{M}$ and width $W_{M}$ is coupled electromagnetically to the rectangular SRR element of dimensions $L_{R} \times W_{R}$, width $t$, and gap $W_{g}$. The coupling distance between the monopole and the resonator is denoted by $d_{1}$, whereas the distance separating the ground plane to the lower edge of the SRR is $d_{2}$. The monopole is designed to operate at $2.45 \mathrm{GHz}$. Two configurations were considered depending on the location of the SRR-slot as shown in Figure 2. Details on the parameters design are given in Figure 2(a) and Table 1.

The antenna excitation is ensured by the monopole, which is soldered to the feeding coaxial cable via an sma connector, whereas the SRR acts as a parasitic element. At lower frequency, the monopole acts as a capacitive element and the SRR acts as an inductive one. The presence of the SRR element close to the monopole provides a reactance compensation and consequently we obtain a new resonant frequency lower than the monopole one, which allows the miniaturization of the structure.

\section{Results and Discussion}

The main simulated and measured results of the antenna with its two configurations were presented and discussed in this section, where analysis of the gain, directivity, surface current distribution, and the shape of radiation patterns were done in order to explain the origin of the antenna superdirectivity.

3.1. Simulation. The simulated return loss of the antenna with its two configurations A1 and A2 is plotted in Figure 3.

We can notice that the proposed structure presents a dual-band frequency for both configurations A1 and A2. The higher resonant frequency $F_{M}=2.45 \mathrm{GHz}$ corresponds to the monopole and seems to be unaffected by the presence of the loop resonator.

The lower resonant frequency appears when we add the SRR element in the vicinity of the monopole. This resonance shifts toward low frequencies due to the effect of the parasitic element $\left(F_{R 1}=1.55 \mathrm{GHz}\right.$ for $\mathrm{A} 1 ; F_{R 2}=1.75 \mathrm{GHz}$ for $\left.\mathrm{A} 2\right)$, allowing the size reduction of the structure. 


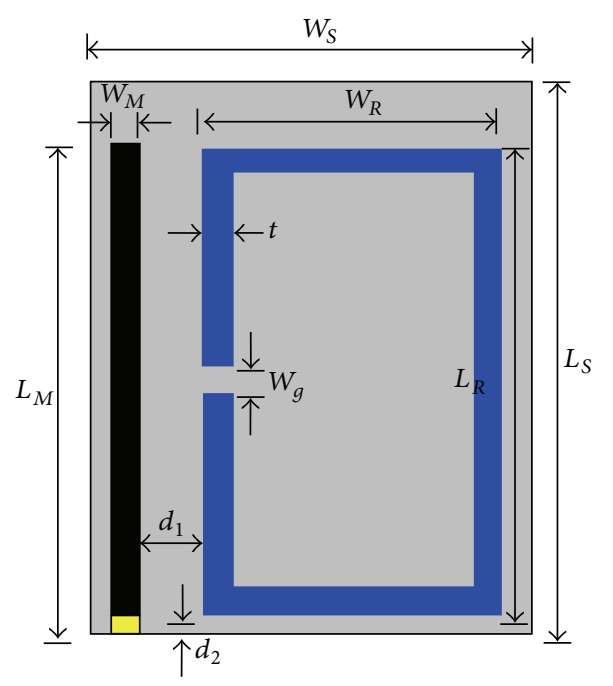

(a)

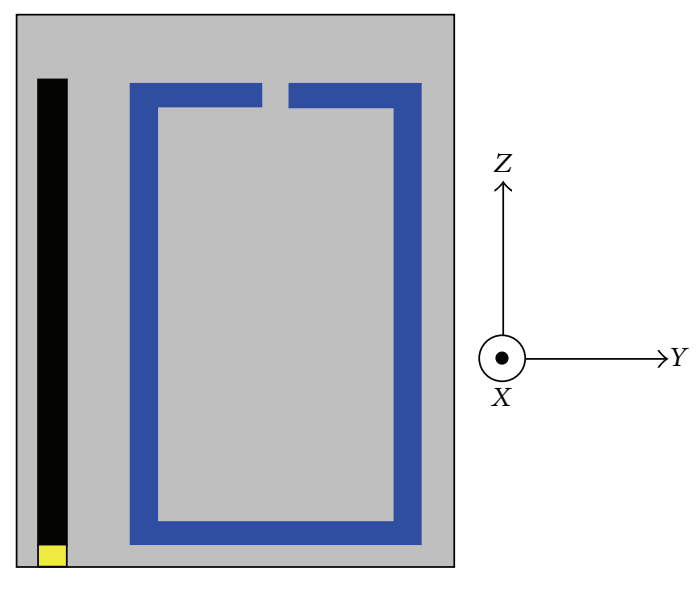

(b)

FiguRE 2: Geometry of the antenna: (a) configuration A1, (b) configuration A2.

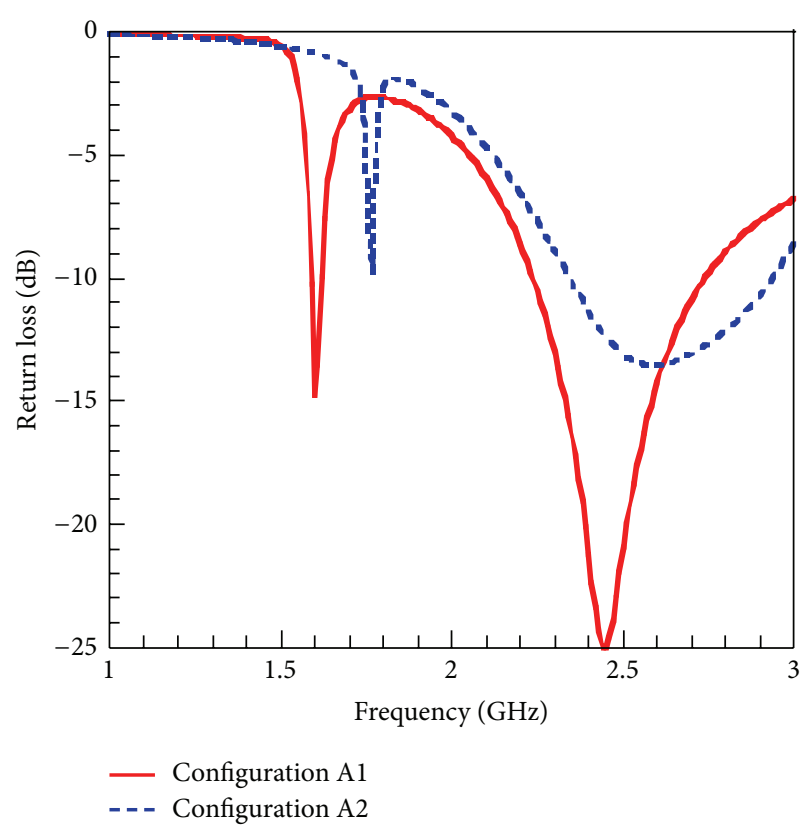

Figure 3: Simulated return loss of the proposed antennas.

However, both resonant frequencies seem to be slightly dependent on the SRR-slot position; a frequency shift of $200 \mathrm{MHz}$ can be noticed between configurations A1 and A2.

We can deduce from Figure 3 that configuration A1 offers more compactness for the structure and better impedance matching at the two operating frequencies than A2.

According to Figure 4(a), when the frequency $F<$ $F_{M}$, the imaginary part of the input impedance is negative; the monopole acts then as a capacitive element in this frequency range. However, when the frequency $F<$ $F_{R}$, the imaginary part of the input impedance is positive (Figures 4(b) and 4(c)) and the SRR acts as an inductive element.
TABle 1: Parameters design.

\begin{tabular}{lc}
\hline & Dimensions in $\mathrm{mm}$ \\
Parameters & Values \\
\hline$L_{S}$ & 28 \\
$W_{S}$ & 24 \\
$L_{M}$ & 26.5 \\
$W_{M}$ & 1.5 \\
$L_{R}$ & 25.5 \\
$W_{R}$ & 17 \\
$t$ & 1.5 \\
$W_{g}$ & 1.5 \\
$d_{1}$ & 5 \\
$d_{2}$ & 1 \\
\hline
\end{tabular}

Consequently, the effect of the monopole is compensated by the SRR, and the input impedance of the structure is achieved at the lower resonance frequency $F_{R}$, which improves the compactness of the structure.

During the numerical analysis and parametric optimization of the antenna, we have found that the main parameter that may affect the antenna radiation is the coupling distance between the monopole and the RSS element. We have studied then the directivity and the gain as a function of the distance $d_{1}$ between the monopole and the SRR for the two configurations of the antenna (Figure 5).

We can notice first from Figure 5 that both of the directivity and the gain of the structure depend on the coupling distance between the resonator and the monopole.

According to Figure 5(a), the maximum simulated directivities are $8.2 \mathrm{dBi}$ and $9.2 \mathrm{dBi}$ for configurations $\mathrm{Al}$ and A2, respectively. We should note here that these values were obtained for coupling distances $d_{1}=20 \mathrm{~mm}$ for A1 and $d_{1}=4 \mathrm{~mm}$ for $\mathrm{A} 2$, where the antenna is not well matched. In fact, the best impedance matching is obtained 


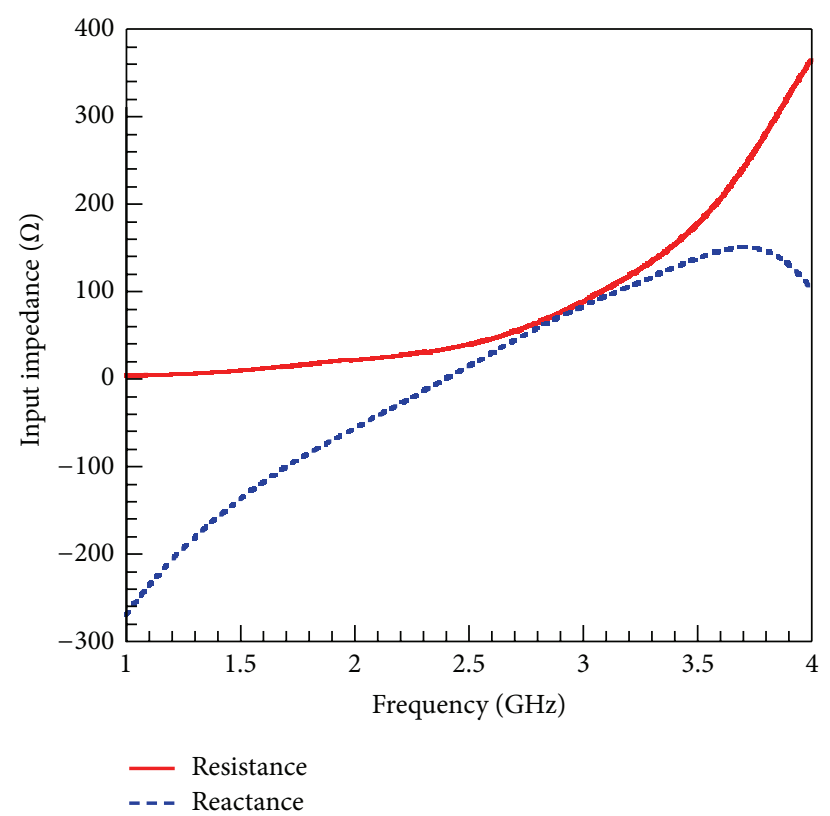

(a)

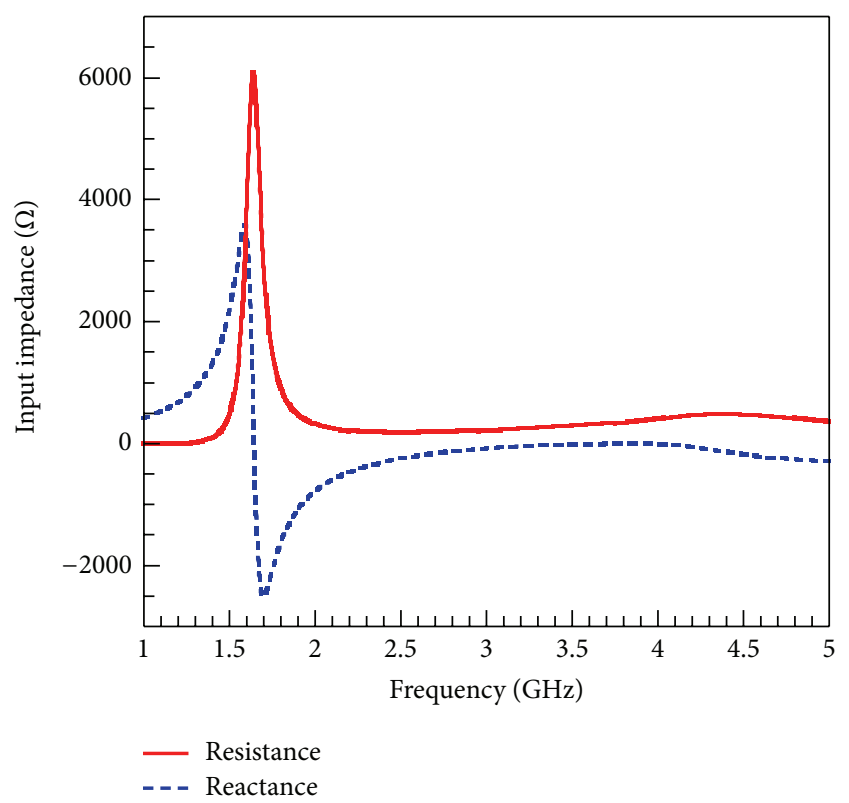

(b)

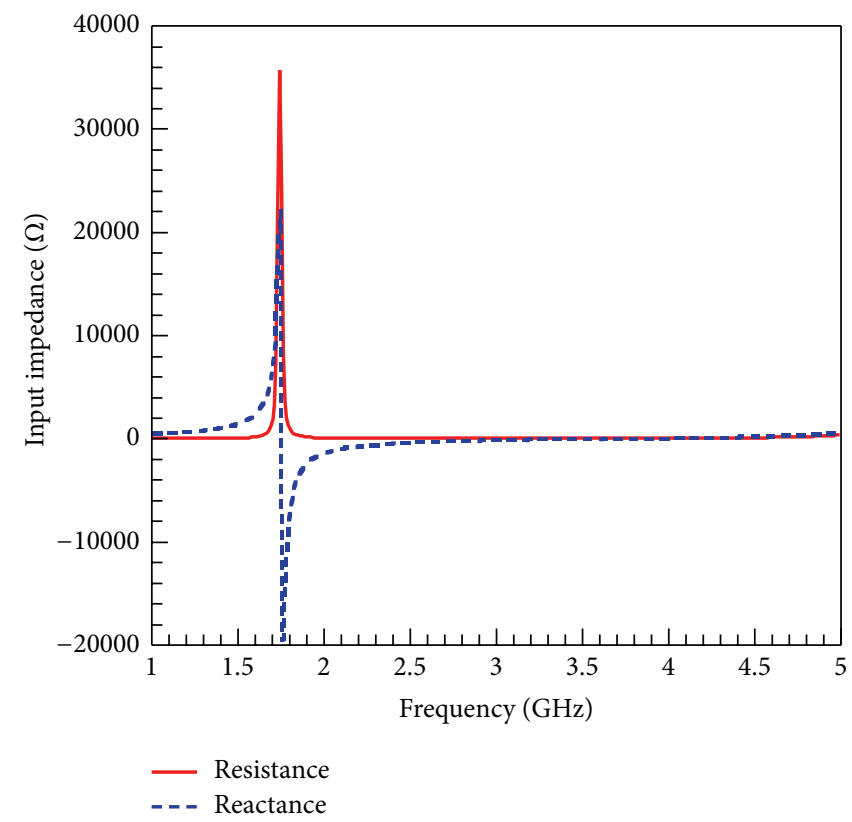

(c)

FIGURE 4: Simulated complex input impedance of the (a) monopole alone; (b) SRR alone (configuration A1); (c) SRR alone (configuration A2).

for coupling distances equal to $4 \mathrm{~mm}$ and $5 \mathrm{~mm}$ for $\mathrm{Al}$ and A2, respectively. However at these distances the maximum directivity drops to $4.41 \mathrm{dBi}$ for both configurations.

For the simulated gain, we can notice from Figure 5(b) that the maximum values are about $9 \mathrm{dBi}$ and $7 \mathrm{dBi}$, for $\mathrm{A} 1$ and $\mathrm{A} 2$, respectively. These values were also obtained for coupling distances $\mathrm{d} 1$ different from those optimized for the best impedance matching. We can also remark that the gain is more stable for configuration A2 than A1 over the coupling distance range $1-12 \mathrm{~mm}$.
In Figure 6, we have considered the chart established in [22] to classify the antenna directivity versus the size of three antennas reported in the literature, and we have placed our simulated maximum values obtained for configurations A1 and A2. Harrington's limit was calculated by considering (3).

Since the radius of the sphere enclosing the structure is $a=20 \mathrm{~mm}$ for configuration A1 and $a=22.6 \mathrm{~mm}$ for A2, at the lowest resonance frequencies $F_{1 R}=1.55 \mathrm{GHz}$ and $F_{2 R}=$ $1.75 \mathrm{GHz}$, respectively, then $k a \sim 0.65$ and $2 a / \lambda=0.20$ for A1, while $k a \sim 0.78$ and $2 a / \lambda=0.24$ for A2. The corresponding 


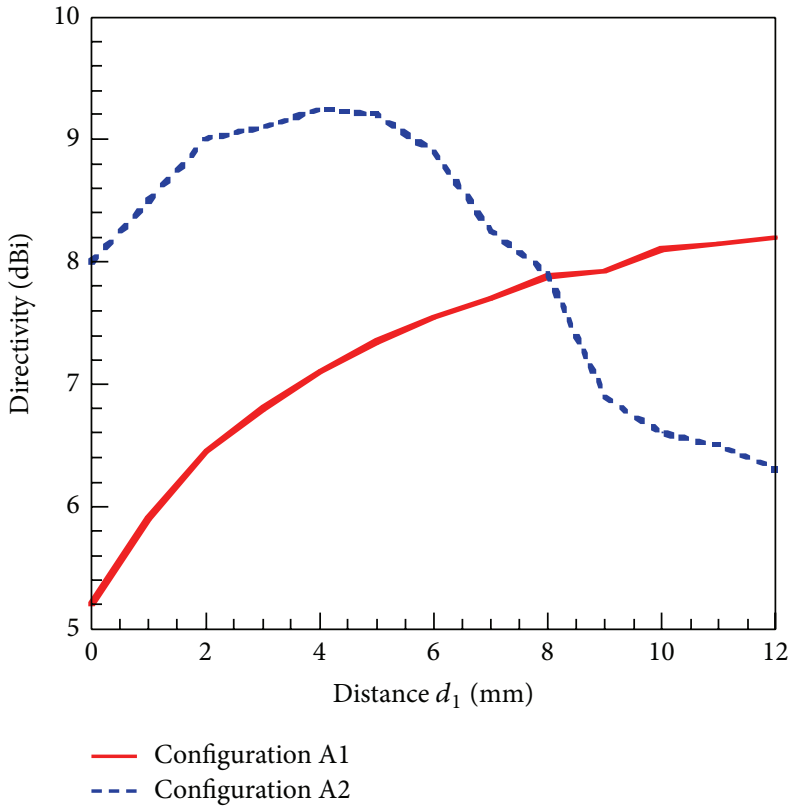

(a)

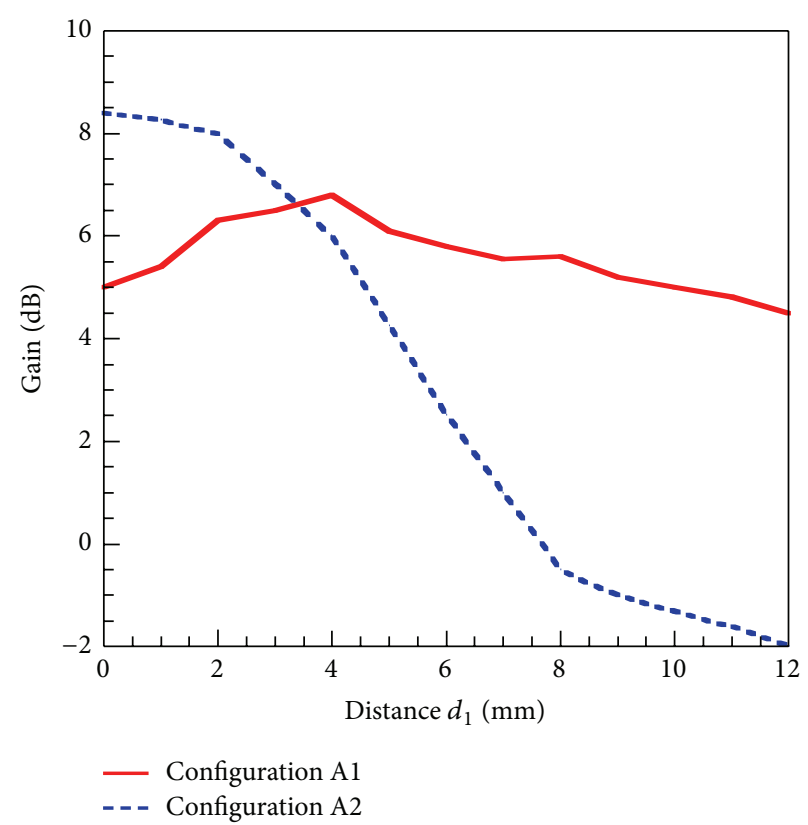

(b)

Figure 5: Effect of the coupling distance $d_{1}$ at the frequency $F_{R}$, on the simulated directivity $(\mathrm{a})$ and gain $(\mathrm{b})$, for configurations $\mathrm{Al}\left(F_{R}=\right.$ $1.55 \mathrm{GHz})$ and $\mathrm{A} 2\left(F_{R}=1.75 \mathrm{GHz}\right)$.

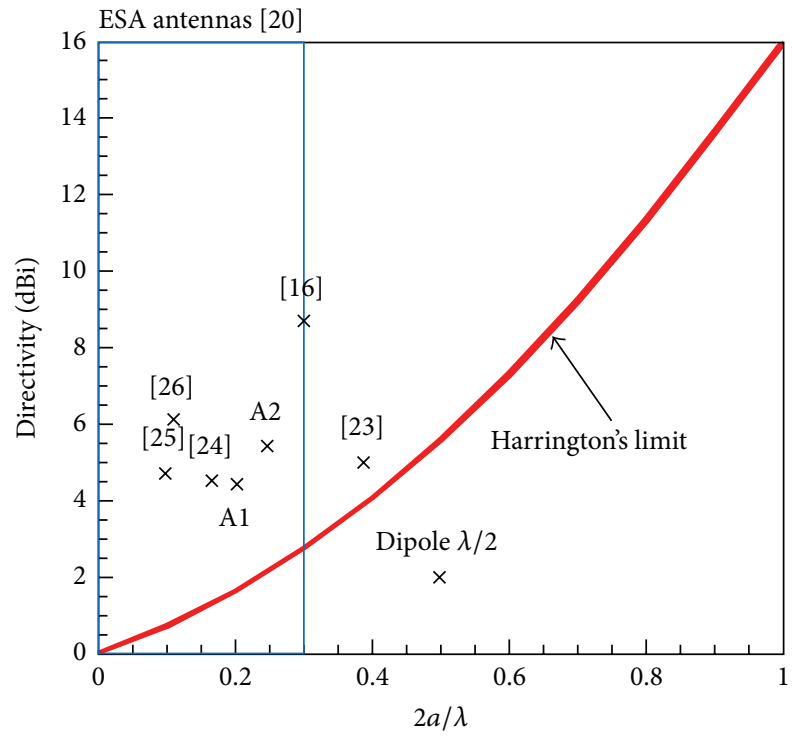

Figure 6: Position of simulated maximum directivities of the structure with its two configurations $\mathrm{A} 1$ and $\mathrm{A} 2$ in the chart representing the directivity versus the antenna size.

values of the directivity $(4.41 \mathrm{dBi}$ for $\mathrm{A} 1$ and $5.5 \mathrm{dBi}$ for A2) are placed in the chart of Figure 6. These values which are calculated from the maximum directivities presented in Figure 5(a) by subtracting $3 \mathrm{dBi}$ (in order to remove the effect of the ground plane) are situated in the region characterizing ESA antennas above Harrington's directivity limit. Consequently, both configurations $\mathrm{A} 1$ and $\mathrm{A} 2$ of the studied structure are situated in the first zone of the chart dedicated to small antennas and above Harrington's limit, which means that the proposed structure can be considered as a superdirective and compact antenna.

The superdirectivity of the antenna may be attributed to the constructive interference between radiated fields from both the monopole and the resonator; these fields should be in phase or phase-opposition and with the same order of magnitude to obtain the highest directivity.

By analyzing the current distribution for configuration A1 (Figure 7(a)) at the lower operating frequency $F_{R}$ and for the optimized coupling distance $d_{1}$, we can remark that the current vectors on the horizontal arms of the SRR element are weak and opposite which leads to their compensation. In addition, these currents are orthogonal to the monopole's current, which may lead to a destructive superposition between radiated fields from the monopole and the resonator. However, the current vector on the monopole is high and concentrated only on the right vertical arm of the SRR. This current vector is parallel and opposite to the monopole's current (phase-opposition), which leads to a constructive superposition between radiated fields and then increases of the antenna directivity.

For configuration A2 (Figure 7(b)), in addition to the vertical SRR current which, as for configuration $\mathrm{Al}$, is parallel to the monopole's current and may explain the increase of directivity, the horizontal SRR current is localized only on the inferior arm of the resonator, close to the ground plane. This high coupling SRR-ground plane may explain the further increase of the directivity of configuration A2 compared to A1. 


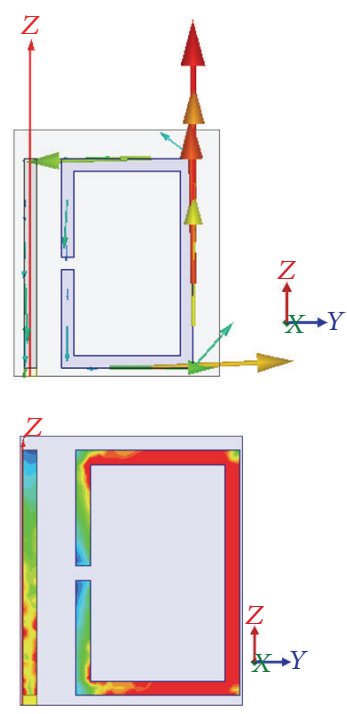

(a)

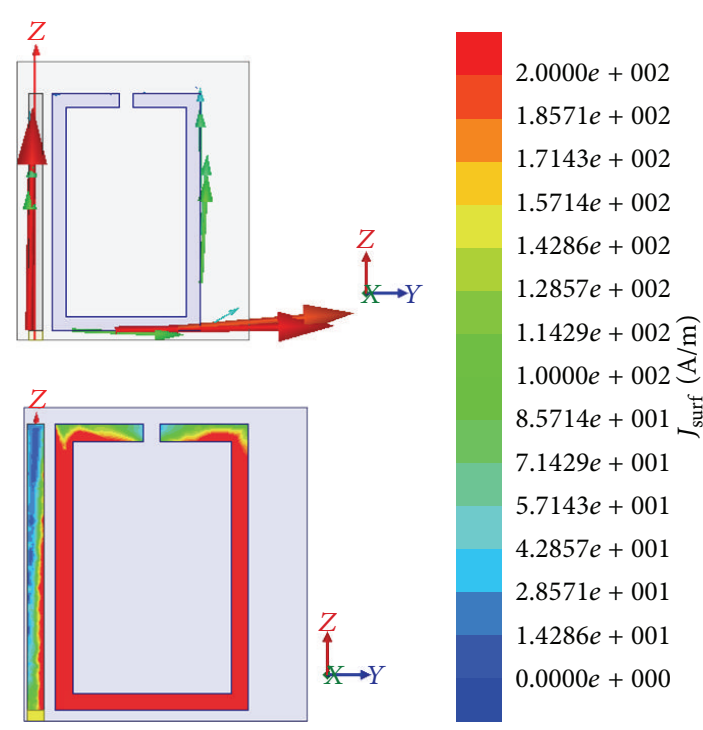

(b)

FIGURE 7: Simulated vector (up) and scalar (down) currents on the monopole and the SRR at the operating frequency $F_{R}$ and for the coupling distance $d_{1}$ : (a) configuration $\mathrm{Al}\left(F_{R}=1.55 \mathrm{GHz}, d_{1}=4 \mathrm{~mm}\right)$; (b) configuration $\mathrm{A} 2\left(F_{R}=1.75 \mathrm{GHz}, d_{1}=1 \mathrm{~mm}\right)$.

In order to confirm our hypothesis, we have plotted in Figure 8 the current magnitude on both the monopole and the SRR element for different coupling distances.

According to Figure 7(a) dealing with configuration A1, the current magnitudes on the monopole and the SRR are similar (for $d_{1}=4 \mathrm{~mm}, J_{s} \sim 2.2 \cdot 10^{2} \mathrm{~A} / \mathrm{m}$ ) for configuration A1, while, for configuration A2 (Figure 7(b)), they are different (for $d_{1}=4 \mathrm{~mm}, J_{s} \sim 1.6 \cdot 10^{2} \mathrm{~A} / \mathrm{m}$ for the monopole and $J_{s} \sim 3.4 \cdot 10^{2} \mathrm{~A} / \mathrm{m}$ for the SRR). We should note here that by considering coupling distance values slightly superior ( 5 or $6 \mathrm{~mm}$ ) to the considered value $d_{1}=4 \mathrm{~mm}$, which gives also high directivities as shown in Figure 5, the discrepancy between the monopole and SRR currents is clearly reduced.

For configuration A2 (Figure 7(b)), although, for the coupling distance $d_{1}=1 \mathrm{~mm}$, the currents on both the monopole and the SRR are different, the effect of the SRRground plane coupling $\left(d_{2}=1 \mathrm{~mm}\right)$ seems to be so high to compensate first for the low monopole-SRR coupling and then to improve the total directivity of the structure.

The simulated 3D-radiation patterns of the structure, with a ground plane of dimension $100 \times 100 \mathrm{~mm}^{2}$, are given in Figure 9. These patterns correspond to resonant frequencies $1,55 \mathrm{GHz}$ and $1,75 \mathrm{GHz}$, for configurations $\mathrm{A} 1$ and A2, respectively. We can notice from Figure 9 that the effect of the SRR-slot location on the radiation pattern is not significant.

In addition, the shape of the radiation pattern may be explained by a superposition of two patterns corresponding to an electric dipole (along $z$-axis) and a magnetic dipole (placed orthogonally to $x$-axis). This assumption, verified from simulation of the SRR and the monopole separately, may explain the absence of the radiation null along $z$-axis for the structure.

Then, we can suppose that the structure behaves as a Huygens source, where the SRR element acts as a magnetic dipole and the monopole as electric dipole, which may explain the antenna superdirectivity. The ground plane contributes also to reflect the radiated power from the antenna toward the upper half-plane $(z>0)$, which increases further the directivity.

3.2. Experimental Validation. The two prototypes of the structure corresponding to configurations $\mathrm{A} 1$ and A2 (see Figure 10) were realized and characterized by measuring their impedance matching, radiation patterns, and directivity. The return losses were measured over the frequency range $1-3 \mathrm{GHz}$ by using an Agilent-N5230A network analyzer, whereas the radiation patterns and directivity measurements were carried out in the anechoic chamber "SATIMO Stargate32" situated at the IETR Institute.

The measured return losses of the designed antenna for configurations $\mathrm{A} 1$ and $\mathrm{A} 2$ are given in Figure 11. We can notice from this figure a good agreement between simulation and measurement with a small discrepancy due to fabrication errors.

As shown in Figure 11, the proposed antenna presents a dual-band behaviour. The main operating frequency of the structure is the lowest one. Then, this resonant frequency $F_{R}$ is equal to $1.55 \mathrm{GHz}$ for $\mathrm{A} 1$ and $1.75 \mathrm{GHz}$ for $\mathrm{A} 2$. The impedance matching of the structure can be better improved by adjusting the distance $d_{2}$ separating the SRR element from the finite ground plane.

Table 2 resumes simulated and measured overall efficiencies (OE) for the two proposed antennas at both lower and higher operating frequencies. The overall efficiency $\mathrm{OE}$ is defined as the ratio of the radiated power $P_{\text {rad }}$ to the input power $P_{\text {in }}$ [31]:

$$
\mathrm{OE}=\frac{P_{\mathrm{rad}}}{P_{\text {in }}}
$$




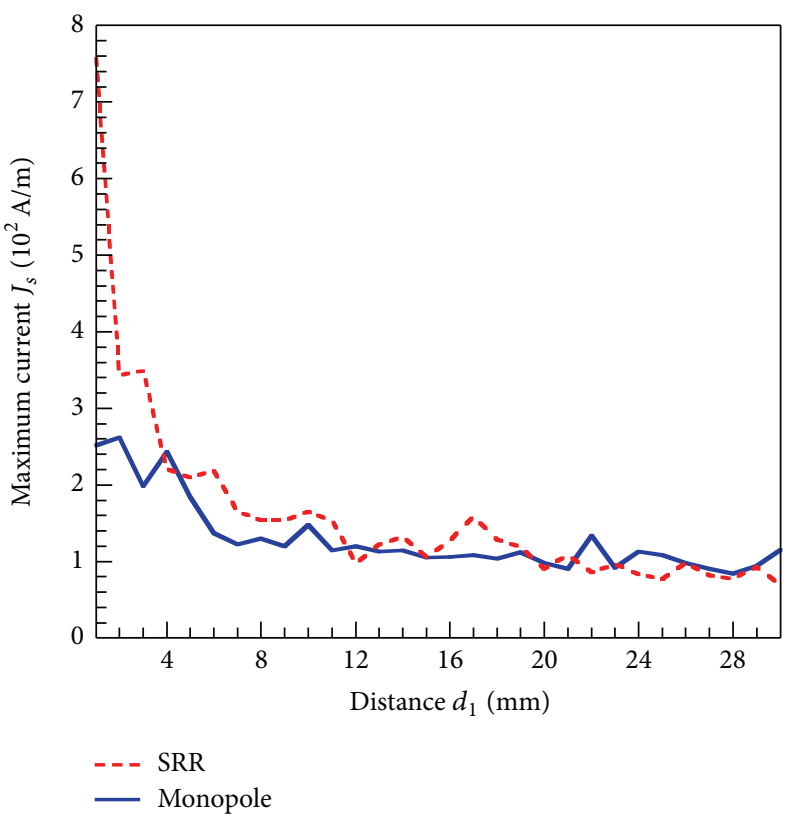

(a)

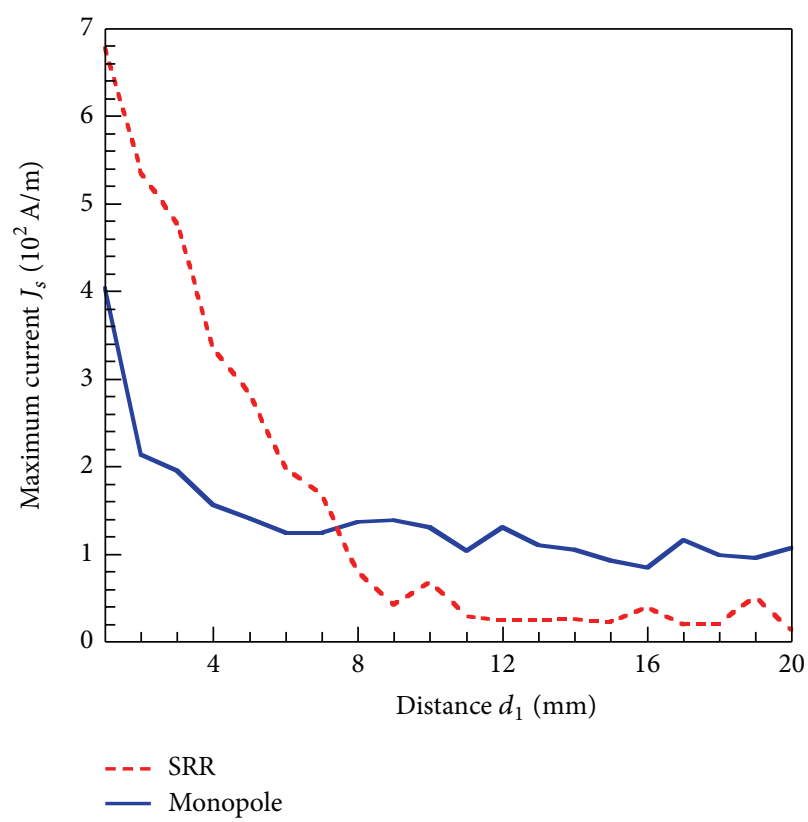

(b)

Figure 8: Maximum current $J_{S}(\mathrm{~A} / \mathrm{m})$, at the operating frequency $F_{R}$, on the monopole, and the SRR versus the coupling distance $d_{1}$ : (a) configuration $\mathrm{A} 1\left(F_{R}=1.55 \mathrm{GHz}\right)$, (b) configuration A2 $\left(F_{R}=1.75 \mathrm{GHz}\right)$.

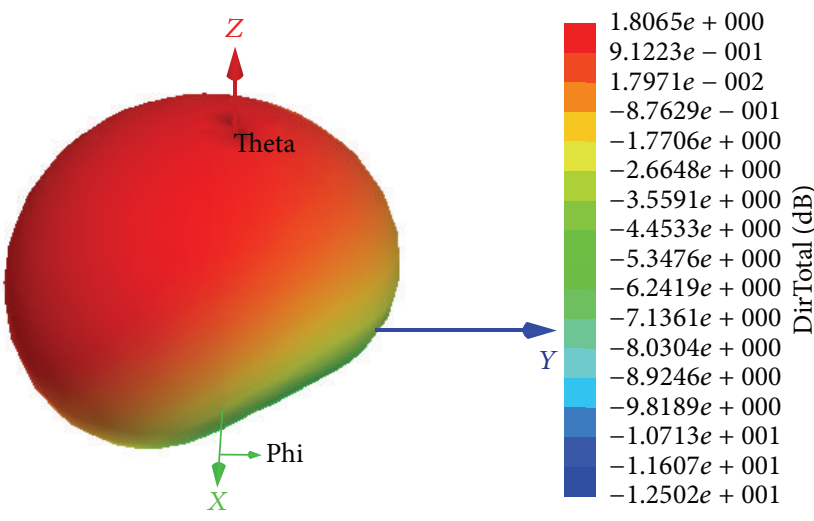

(a)

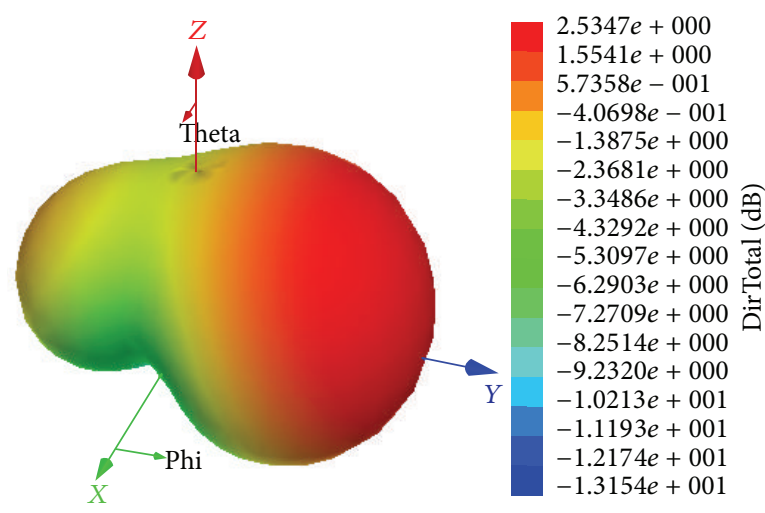

(b)

FIgURE 9: Simulated 3D-radiation patterns for the structure: (a) configuration A1 ( $\left.F_{R}=1.55 \mathrm{GHz}\right)$, (b) configuration A2 $\left(F_{R}=1.75 \mathrm{GHz}\right)$.

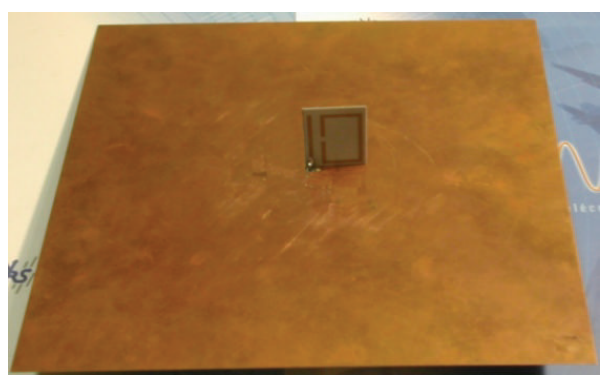

(a)

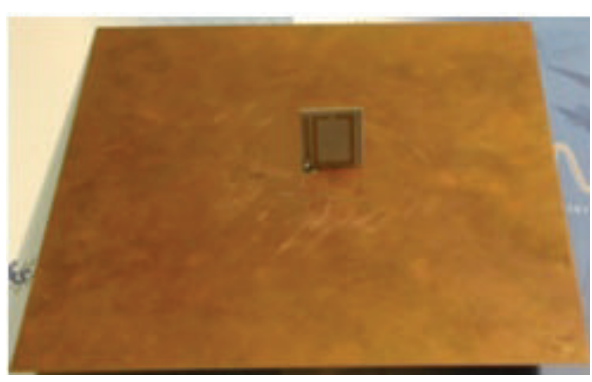

(b)

FIgURE 10: Photos of the realized prototypes for the designed antenna: (a) configuration A1; (b) configuration A2. 


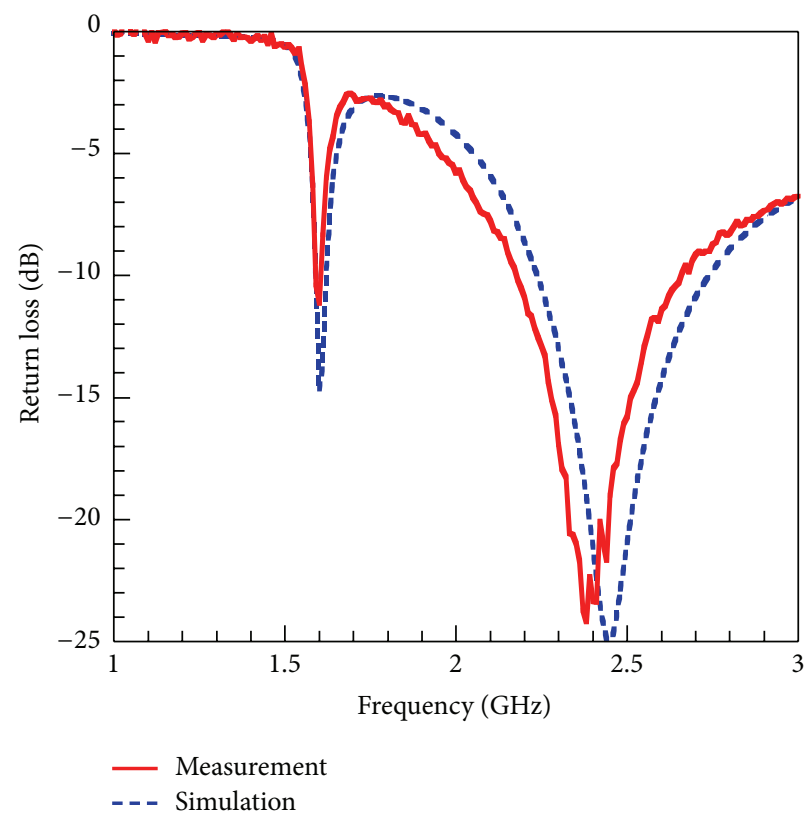

(a)

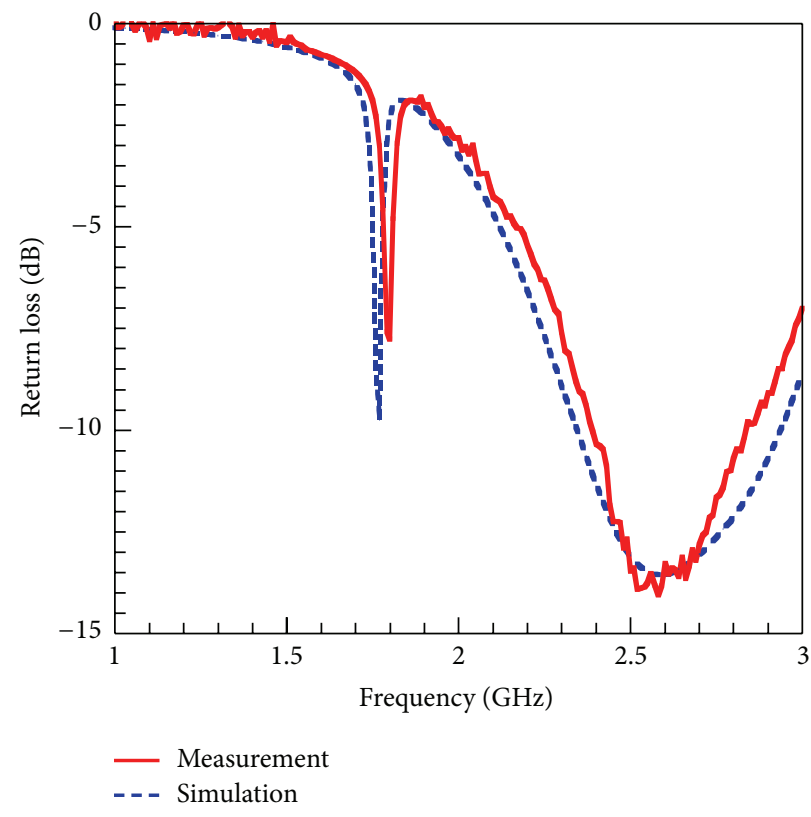

(b)

FIGURE 11: Comparison between simulated and measured return losses for the designed antenna with a coupling distance $d_{1}$ : (a) configuration $\mathrm{A} 1\left(d_{1}=4 \mathrm{~mm}\right)$; (b) configuration A2 $\left(d_{1}=1 \mathrm{~mm}\right)$.

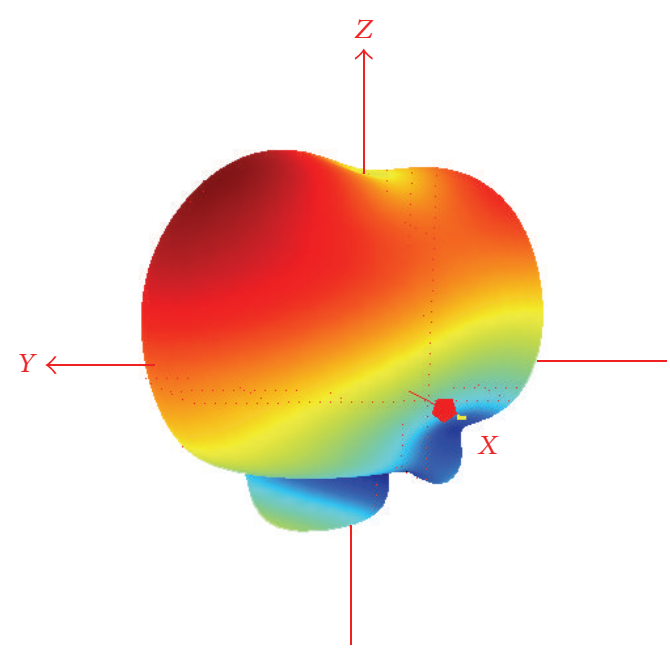

(a)

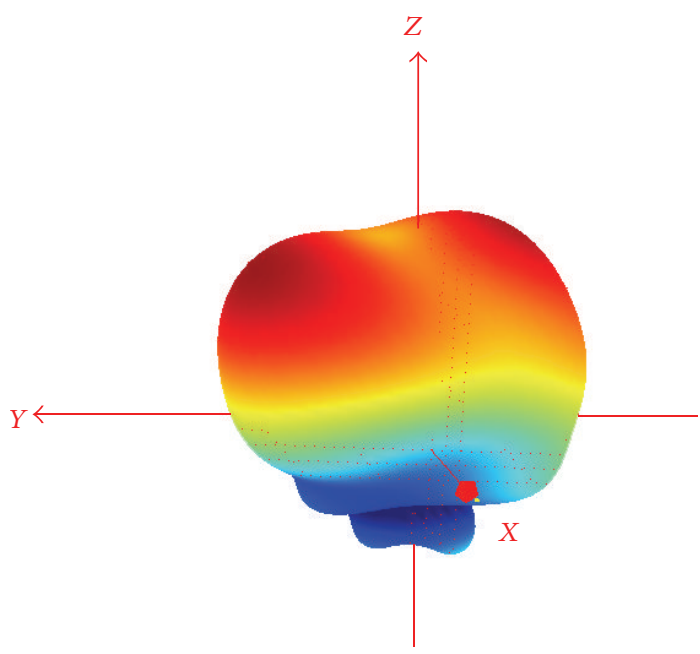

(b)

FIGURE 12: Measured 3D radiation patterns of the studied antenna at the operating frequency $F_{R}$ and for the coupling distance $d_{1}$ : (a) configuration $\mathrm{A} 1\left(F_{R}=1.55 \mathrm{GHz}, d_{1}=4 \mathrm{~mm}\right)$, (b) configuration $\mathrm{A} 2\left(F_{R}=1.75 \mathrm{GHz}, d_{1}=1 \mathrm{~mm}\right)$.

TABLE 2: Simulated and measured overall efficency (OE) for the two proposed antennas.

\begin{tabular}{lcccc}
\hline \multirow{2}{*}{ Antenna } & \multicolumn{2}{c}{ Simulated overall efficiency $(\mathrm{OE} \%)$} & \multicolumn{2}{c}{ Measured overall efficiency (OE \%) } \\
& $f_{0}$ & $f_{1}$ & $f_{0}$ & $f_{1}$ \\
\hline A1 & 96.2 & 95.8 & 72.3 & 82.6 \\
A2 & 94.8 & 88.8 & 82.1 & 80.4
\end{tabular}


TABLE 3: Performance comparison of three electrical small metamaterial-inspired antennas.

\begin{tabular}{lcccc}
\hline & $F_{R}(\mathrm{MHz})$ & $k a$ & Measured OE (\%) & Measured gain $(\mathrm{dB})$ \\
\hline Antenna A1 & 1537 & 0.58 & 72.3 & 4.28 \\
Z-antenna [32] & 1575 & 0.44 & 87.6 & 3.23 \\
Egyptian-axe dipole antenna [33] & 1567 & 0.99 & 79.6 & 4.25 \\
\hline
\end{tabular}

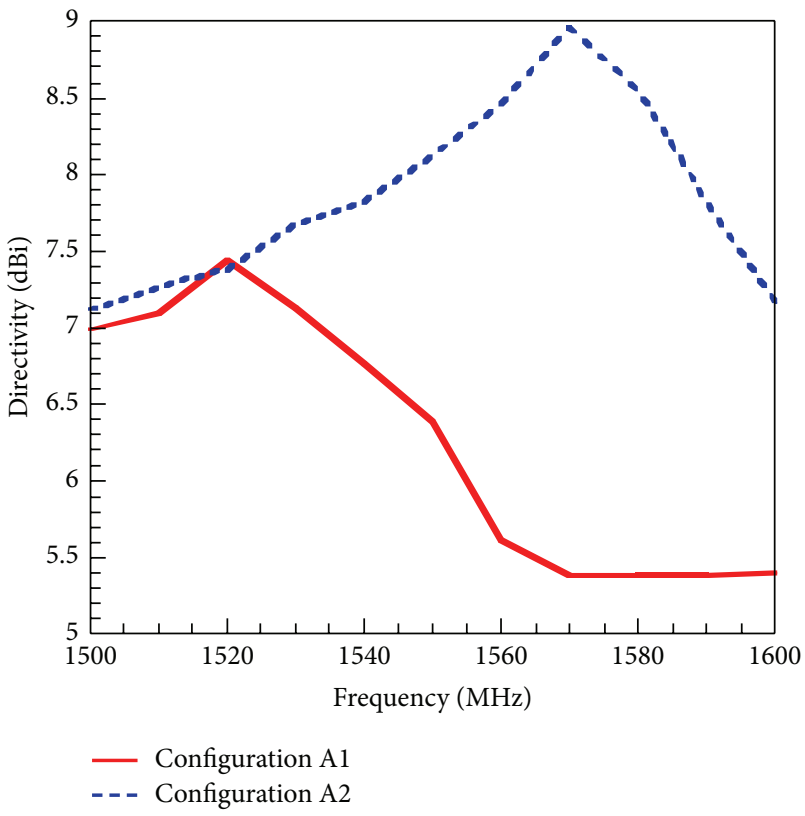

FIGURE 13: Measured directivity of the studied antenna with coupling distance $d_{1}$ : (a) configuration $\mathrm{Al}\left(d_{1}=4 \mathrm{~mm}\right)$, (b) configuration $\mathrm{A} 2\left(d_{1}=1 \mathrm{~mm}\right)$.

By analyzing results from Table 2, we remark that the overall efficiency is usually higher at the lower resonance frequency (except for measured value of $\mathrm{Al}$ ). We should mention, for example, that, for lower frequency of antenna A1, the high value of the overall efficiency $(\mathrm{OE}=96 \%)$ drops clearly when we remove the SRR resonator, which confirms the main role of the metamaterial-cell in the amelioration of the directivity.

The measured radiation patterns of the antenna for both configurations $\mathrm{A} 1$ and $\mathrm{A} 2$ are illustrated in Figure 12. We can remark that the antenna focuses its radiation in the halfspace situated above the ground plane. This behaviour is due to the coupling effect between the monopole and the SRR in addition to the presence of the ground plane. We should note that, by increasing the size of the ground plane, we may improve the directivity of the proposed antennas by minimizing the back radiation.

As shown in Figure 13, the maximum measured directivity of the antenna is around $7.5 \mathrm{dBi}$ for configuration $\mathrm{A} 1$ and $9 \mathrm{dBi}$ for A2. As predicted by simulation in Figure 5(a), the configuration A2 seems to be more directive than A1. This may be explained by the ground plane coupling effect which is more important in configuration $\mathrm{A} 1$ than $\mathrm{A} 2$.

The improvement of the antenna directivity is due essentially to the coupling effect between the monopole and the SRR which affects the surface currents in the structure and then the whole antenna radiation behaviour, especially its gain and directivity.

In order to better evaluate the performances of the optimized structure A1, we have compared it to two similar antennas $[32,33]$ in terms of size $(k a)$, efficiency $(\mathrm{OE})$, and gain. Table 3 regroups the obtained results.

The "Z-antenna" is a metamaterial-inspired structure that incorporates a lumped inductor element of value $19 \mathrm{nH}$ to reduce its height [32], whereas the Egyptian-axe dipole antenna [33] uses an electromagnetic band-gap (EBG) structure, which acts as an artificial magnetic conductor ground plane (AMC), in order to increase the gain.

By comparing the optimised antenna Al to similar structures developed in $[32,33]$, we can situate it, in terms of performances, between the Z-antenna and the Egyptian-axe dipole antenna. We have improved the gain $(4.28 \mathrm{~dB}$ against $3.23 \mathrm{~dB}$ for the $\mathrm{Z}$-antenna) and the size $(k a=0.58$ against 0.99 for the Egyptian-axe dipole antenna). Therefore, the directivity was slightly decreased but still high compared to conventional patch antennas.

\section{Conclusion}

The deep directivity analysis of a metamaterial-inspired compact monopole antenna was realized. It is shown that the proposed structure (SRR + monopole) with its two configurations $\mathrm{A} 1$ and $\mathrm{A} 2$ is compact $\left(0.145 \lambda_{0}\right)$ and may exhibit a superdirectivity behaviour. From the analysis of simulated currents distribution and shape of radiation patterns, we have proposed that the superdirectivity of the structure is due especially to the monopole-SRR coupling $\left(D_{\max }=7.5 \mathrm{dBi}\right.$ for A1). This directivity may be improved further by optimizing also the ground plane-SRR coupling $\left(D_{\max }=9 \mathrm{dBi}\right.$ for A2).

\section{Conflict of Interests}

The authors declare that there is no conflict of interests regarding the publication of this paper.

\section{Acknowledgment}

This paper was funded by the Deanship of Scientific Research (DSR), King Abdulaziz University, Jeddah. The authors, therefore, acknowledge with thanks DSR technical and financial support.

\section{References}

[1] K. L. Wong, Compact and Broadband Microstrip Antennas, John Wiley \& Sons, 2004. 
[2] R. C. Hansen, Electrically Small Superdirective and Superconducting Antennas, John Wiley \& Sons, New York, NY, USA, 2006.

[3] J. M. Laheurte, Compact Antennas for Wireless Communications and Terminals: Theory and Design, John Wiley \& Sons, 2013.

[4] R. W. Ziolkowski, P. Jin, and C.-C. Lin, "Metamaterial-inspired engineering of antennas," Proceedings of the IEEE, vol. 99, no. 10, pp. 1720-1731, 2011.

[5] A. Erentok and R. W. Ziolkowski, "A dual-band efficient metamaterial-inspired electrically-small magnetic-based antenna," in Proceedings of the IEEE Antennas and Propagation Society International Symposium, pp. 1877-1880, Honolulu, Hawaii, USA, June 2007.

[6] P. Jin and R. W. Ziolkowski, "Broadband, efficient, electrically small metamaterial-inspired antennas facilitated by active nearfield resonant parasitic elements," IEEE Transactions on Antennas and Propagation, vol. 58, no. 2, pp. 318-327, 2010.

[7] S. Dakhli, K. Mahdjoubi, H. Rmili, J. M. Floch, and H. Zangar, "Compact, multifunctional, metamaterial-inspired monopole antenna," in Proceedings of the 6th European Conference on Antennas and Propagation (EuCAP '12), pp. 1967-1970, Prague, Czech Republic, March 2012.

[8] R. Goncalves, N. B. Carvalho, and P. Pinho, "Metamaterial inspired compact printed antenna for WLAN applications," in Proceedings of the IEEE Antennas and Propagation Society International Symposium (APSURSI '13), pp. 1382-1383, July 2013.

[9] S. Dakhli, J.-M. Floch, K. Mahdjoubi, H. Rmili, and H. Zangar, "Compact and multi-band metamaterial-inspired dipole antenna," in Proceedings of the 7th European Conference on Antennas and Propagation (EuCAP'13), pp. 2765-2768, Gothenburg, Sweden, April 2013.

[10] P. Jin and R. W. Ziolkowski, "Low-Q, electrically small, efficient near-field resonant parasitic antennas," IEEE Transactions on Antennas and Propagation, vol. 57, no. 9, pp. 2548-2563, 2009.

[11] R. W. Ziolkowski, P. Jin, and C.-C. Jin, "Electrically small metamaterial-inspired antennas: designs and measurements, efficiency and bandwidth performance," in Proceedings of the IEEE International Workshop on Antenna Technology (iWAT '09), pp. 1-4, March 2009.

[12] S. Dakhli, K. Mahdjoubi, J.-M. Floch, H. Rmili, and H. Zangar, "Efficient, metamaterial-inspired loop-monopole antenna with shaped radiation pattern," in Proceedings of the Loughborough Antennas and Propagation Conference (LAPC '12), pp. 1-4, Loughborough, UK, November 2012.

[13] M. A. W. Nordin and M. T. Islam, "A bandwidth enhanced printed dipole antenna with metamaterial-inspired loading," in Proceedings of the 3rd IEEE International Conference on Space Science and Communication (IconSpace '13), pp. 182-185, Melaka, Malaysia, July 2013.

[14] M. S. Majedi and A. R. Attari, "A compact and broadband metamaterial-inspired antenna," IEEE Antennas and Wireless Propagation Letters, vol. 12, pp. 345-348, 2013.

[15] N. Zhu, Q. Feng, and Q. Xiang, "Metamaterial-inspired highgain array antenna," in Proceedings of the Cross Strait QuadRegional Radio Science and Wireless Technology Conference (CSQRWC '11), pp. 375-378, July 2011.

[16] A. D. Yaghjian, "Increasing the supergain of electrically small antennas using metamaterials," in Proceedings of the 3rd European Conference on Antennas and Propagation (EuCAP '09), pp. 858-860, Gothenburg, Sweden, March 2009.
[17] H. Mirzaei and G. V. Eleftheriades, "A compact frequencyreconfigurable metamaterial-inspired antenna," IEEE Antennas and Wireless Propagation Letters, vol. 10, pp. 1154-1157, 2011.

[18] O. Turkmen, G. Turhan-Sayan, and R. W. Ziolkowski, "Metamaterial inspired, electrically small, GSM antenna with steerable radiation patterns and high radiation efficiency," in Proceedings of the IEEE Antennas and Propagation Society International Symposium (APSURSI '13), pp. 770-771, Orlando, Fla, USA, July 2013.

[19] J. P. Turpin, J. A. Bossard, K. L. Morgan, D. H. Werner, and P. L. Werner, "Reconfigurable and tunable metamaterials: a review of the theory and applications," International Journal of Antennas and Propagation, vol. 2014, Article ID 429837, 18 pages, 2014.

[20] H. A. Wheeler, "Fundamentals limitations of small antennas," Proceding of the IRE, vol. 35, pp. 1479-1484, 1947.

[21] L. J. Chu, "Physical limitations of omni-directional antennas," Journal of Applied Physics, vol. 19, no. 12, pp. 1163-1175, 1948.

[22] M. Pigeon, L. Rudant, C. Delaveaud, and K. Belmkaddem, "Antennes miniatures superdirectives," in 18ème Journées Nationales microndes (JNM '18), Paris, France, May 2013.

[23] O. Luukkonen, A. O. Karilainen, J. Vehmas, C. Simovski, and S. A. Tretyakov, "high impedance surface based antennas-lose the antenna," in Proceedings of the European Conference on Antennas and Propagation (EUCAP '10), pp. 151-155, Gothenburg, Sweden, April 2010.

[24] P. Jin and R. W. Ziolkowski, "Metamaterial-inspired, electrically small huygens sources," IEEE Antennas and Wireless Propagation Letters, vol. 9, pp. 501-505, 2010.

[25] S. R. Best, "Progress in the design and realization of an electrically small Huygens source," in Proceeding of the International Workshop on Antenna Technology: Small Antennas, Innovative Structures and Materials (iWAT '10), pp. 1-4, Lisbon, Portugal, March 2010.

[26] H. G. Schantz, "Directive, electrically-small UWB antennas," in Proceedings of the IEEE International Conference on UltraWideband (ICUWB '12), pp. 227-231, Syracuse, NY, USA, September 2012.

[27] B. Sentucq, A. Sharaiha, and S. Collardey, "Superdirective metamaterial-inspired electrically small antenna arrays," in Proceedings of the European Conference on Antennas and Propagation (EuCAP '13), pp. 151-155, Gothenburg, Sweden, April 2013.

[28] T. Taylor, "A discussion of the maximum directivity of an antenna," Proceding of the IRE, vol. 36, p. 1135, 1948.

[29] H. Riblet, "Note on the maximum directivity of an antenna," Proceding of the IRE, vol. 36, no. 5, pp. 620-623, 1948.

[30] R. F. Harrington, "On the gain and beamwidth of directional antennas," IRE Transactions on Antennas and Propagation, vol. 6, pp. 219-225, 1958.

[31] A. Erentok and R. W. Ziolkowski, "Metamaterial-inspired efficient electrically small antennas," IEEE Transactions on Antennas and Propagation, vol. 56, no. 3, pp. 691-707, 2008.

[32] P. Jin and R. W. Ziolkowski, "Multiband extensions of the electrically small, near-field resonant parasitic $\mathrm{Z}$ antenna," IET Microwaves, Antennas and Propagation, vol. 4, no. 8, pp. 10161025, 2010.

[33] P. Jin and R. W. Ziolkowski, "High-directivity, electrically small, low-profile near-field resonant parasitic antennas," IEEE Antennas and Wireless Propagation Letters, vol. 11, pp. 305-309, 2012. 

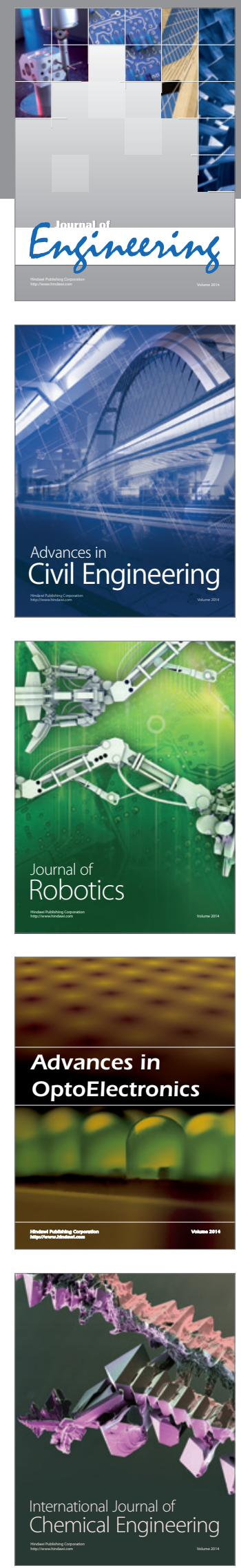

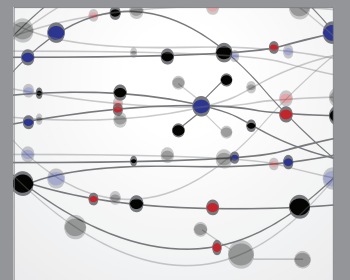

The Scientific World Journal
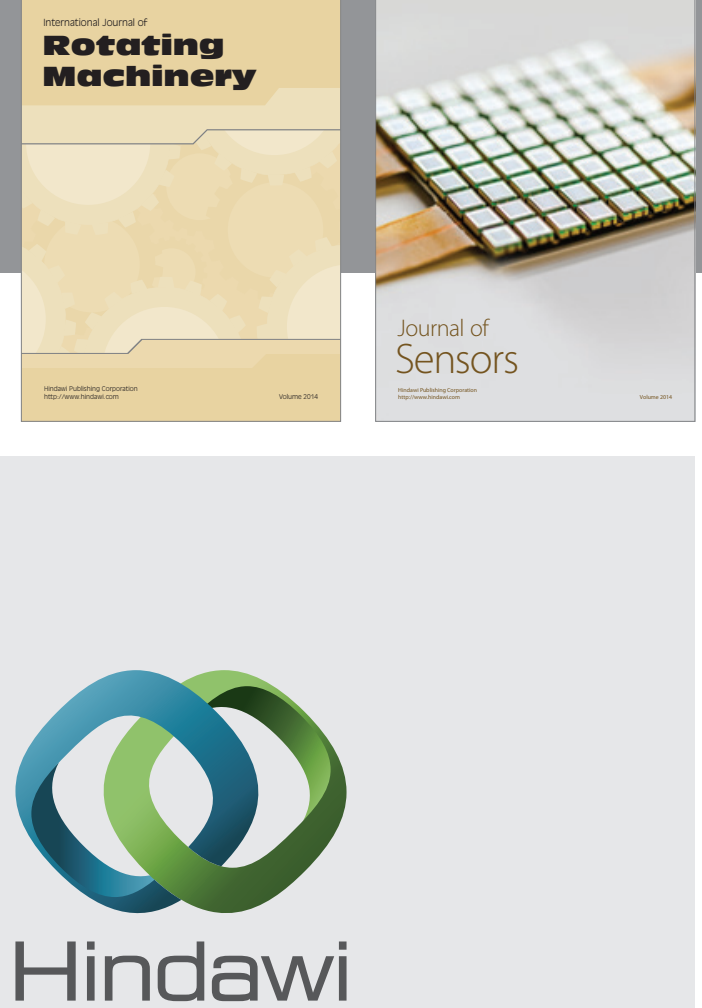

Submit your manuscripts at http://www.hindawi.com
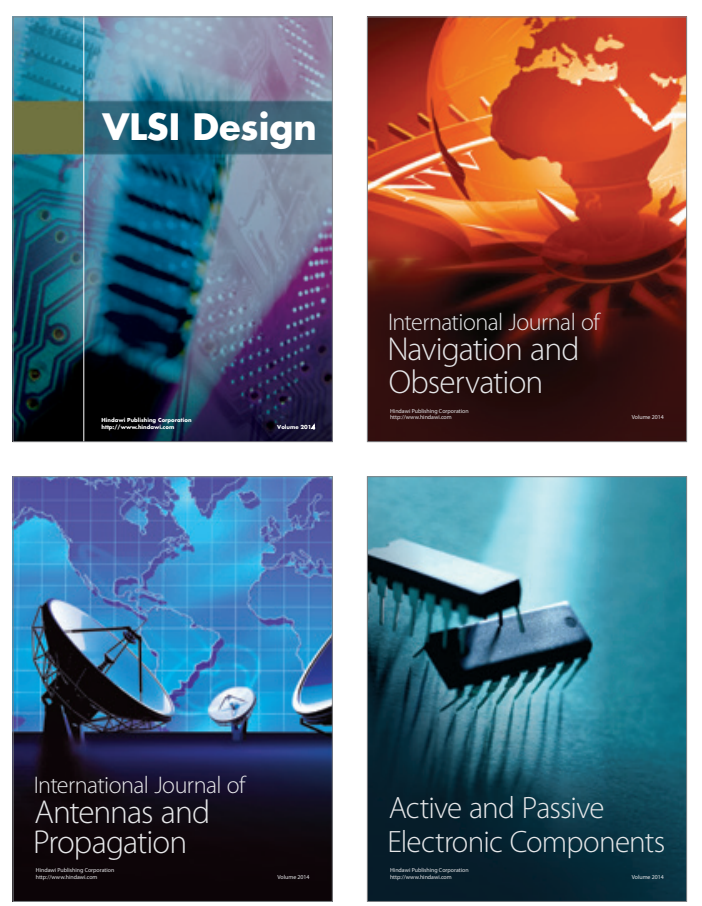
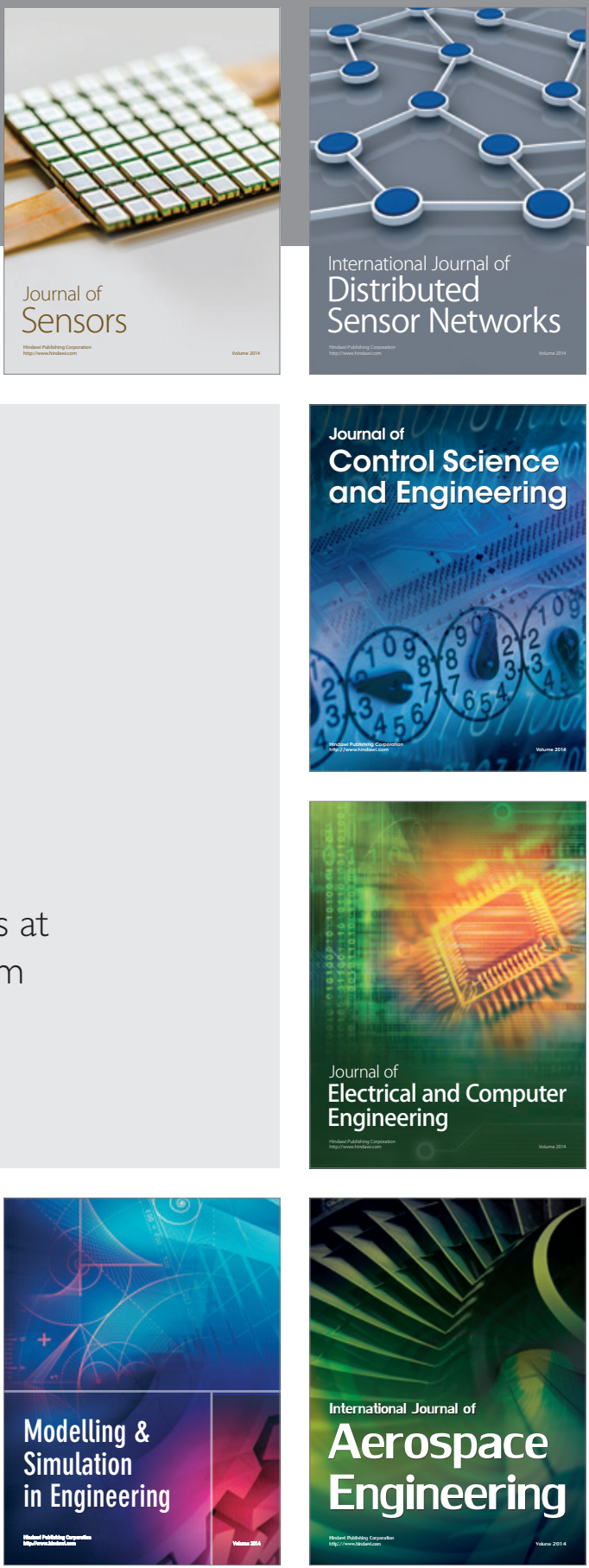

Journal of

Control Science

and Engineering
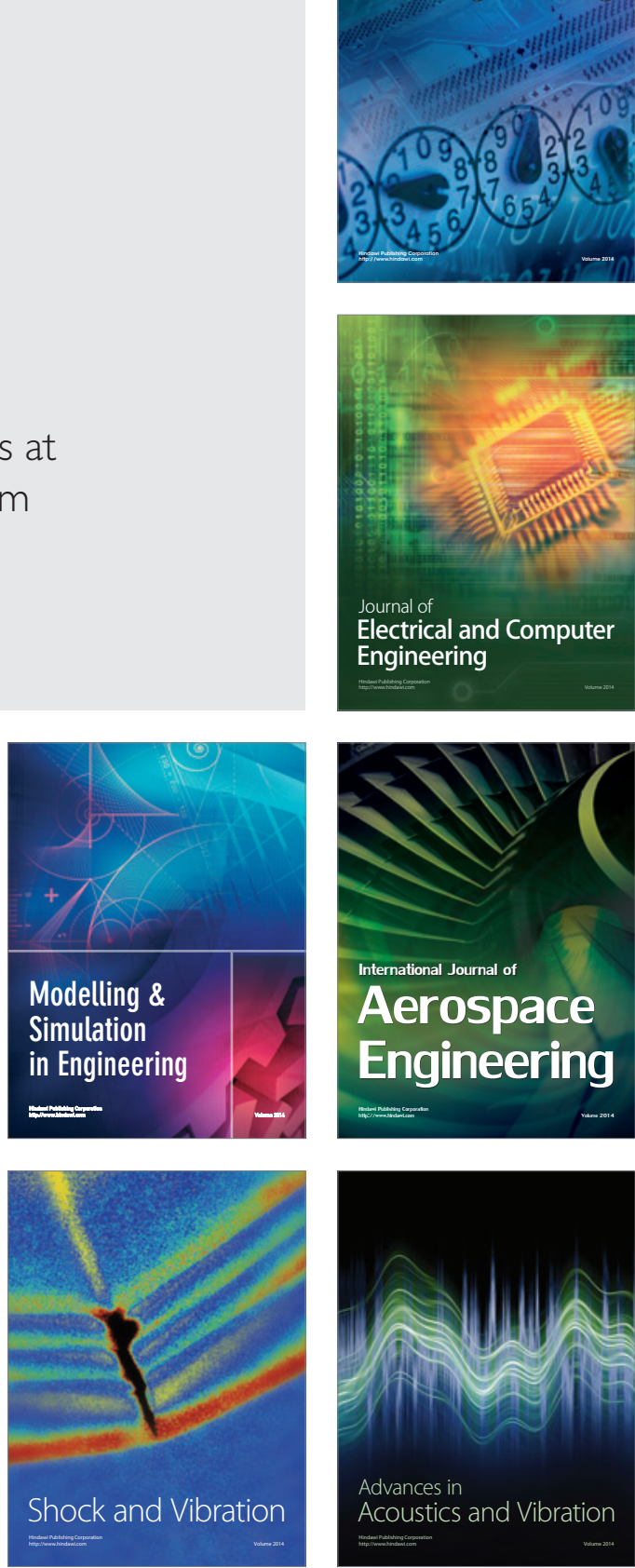